Journal of Theoretical and Applied Mechanics, Sofia, Vol. 47 No. 3 (2017) pp. 3-24

\title{
REGULAR MECHANICAL TRANSFORMATION OF ROTATIONS INTO TRANSLATIONS: PART 2. KINEMATIC S YNTHESIS OF THE ELEMENTS OF HIGH KINEMATIC JOINTS, REALIZING THE PROCESS OF MOTIONS TRANSFORMATION
}

\author{
Emilia Abadjieva $^{1 *}$, Valentin Abadjiev ${ }^{2}$ \\ ${ }^{1}$ Graduate School of Engineering and Resource Science, Faculty of \\ Engineering and Resource Science, Akita University, Tegatagakuen-machi \\ 1-1, Akita, Japan \\ ${ }^{2}$ Institute of Mechanics, Bulgarian Academy of Sciences, Acad. G. Bonchev \\ Str., bl. 4, 1113 Sofia, Bulgaria
}

[Received 13 March 2017. Accepted 26 June 2017]

\begin{abstract}
This work is developed on the basis of the illustrated main parts of the kinematic theory (theory of gearing) of the spatial rack drives in Part 1 of this study. The applied theoretical approach to their synthesis, based on the T. Olivier's second principle is defined. A study of the geometric nature of the surface of action (mesh region, respectively) of these class transmissions is shown. Research software programs for synthesis and visualization of these transmissions and their specific elements are elaborated, on the basis of the given algorithms to the synthesis of the elements of high kinematic joints (active tooth surfaces), with which the movable links of the studied gear systems are equipped.
\end{abstract}

KEY WORDS: Synthesis, mathematical modelling, spatial rack drives, conic linear helicoids.

\section{INTRODUCTION}

The science of gear mechanisms operates with the theoretical apparatus of applied mechanics, which here is oriented to the development of mathematical models, serving analysis and synthesis of the mentioned mechanisms. In the second half of the 20 th century, the accumulated large amount of scientific researches gives impetus to the development of the science of gears towards the formation of two relatively autonomous scientific branches: theory of gearing and geometry of gearing (geometric theory of gear mechanisms) [1-3].

The theory of gearing is a theory of high kinematic joints, taking into account the specifics of their application in gear transmissions. It deals with the common principles, to which the motions transformation are subjected by means of three-links

\footnotetext{
*Corresponding author e-mail: abadjieva@gipc.akita-u.ac.jp
} 
mechanisms with high kinematic joints in all possible placements of the corresponding axes of rotation transformation and directrix of rectilinear translation. The theory of gearing offers an apparatus for analytical and computer study of different types of gearings [4]. In this sense, the obtained basic kinematic-geometric characteristics in "Part 1. Kinematic analysis and a definition of the basic characteristics" of this study extend the theoretical knowledge, which is an object of the theory of gearing.

The geometry of gearing is a geometric theory of the specific types of gear mechanisms. This branch of the gears science occurs as a result of the constantly changing demands of industry to the technological and exploitation properties of mechanical transmissions. Some main tasks of the geometry of gearing are permanently solved, based on the developed analytical apparatus in theory of gearing [4]:

- Choice of rational form and teeth geometry of different types of gear mechanisms;

- Development and scientific justification of the methodology for defining the quality characteristics of conjugated and non-conjugated elements of tooth surfaces (tooth profiles);

- Creation of a specialized computing apparatus for basic and optimization synthesis of new classes of contemporary gears and etc.

The performed survey of the science of the gearing shows that in the specialized literature very limited researches on mechanical gear systems, which are designed to transform rotation into translation motions and vice versa, are presented.

This ascertainment is valid not only for the processes of this type motions transformation, but also for the devices, by which it is realized, and they are known as rack drives. This fact gaves an impact on the implementation of such mechanisms in various branches of the technique: robotics, mechatronics, nanotechnology and so on.

In most known literature sources [5,6], rack gearings are treated as an instrumental gearing, when cylindrical involute gears (straight teeth or helical) are generated (cut). Since the object of this study is a rack drive, as a mechanical motions transformer, here it will be limited to the above comment, relating to the rack instrumental gearing.

The rack mechanical transformers are an object of study by E. Ginzburg, and others in [7]. There, the kinematics of these mechanisms, their technological structure, geometric parameters of rack and cylindrical straight-teeth gear are briefly explained. An algorithm for geometric synthesis of these transmissions is defined and the technological restrictions on geometric parameters, while generating the tooth surfaces of its links, are shown. In the study [7] of M. Watanabe and M. Maki, an analysis and 
manufacturing experiment of rack drive of type Wildhaber-Novikov (WN - helical rack drive) is elaborated. An analysis of the tooth contact and calculation of Hertz contact strength is realized.

In this study, a solution of some parts of the mentioned above tasks of the geometry of gearing is illustrated. It serves the synthesis of spatial rack drives, by using the synthesis of the elements of high kinematics joints, realizing motions transformation of type rotation into translation $(R \leftrightarrow T)$.

\section{COMMON CHARACTERISTICS OF THE GEOMETRY OF ACTION SURFACE OF SPATIAL RACK DRIVE}

\subsection{PRINCIPLES OF T. OlivieR - TERMS AND CHARACTERISTICS}

For all types of gear mechanisms, including rack drives, solving the problem of their kinematic synthesis should give a solution to two groups of tasks, concerning: the geometry and dimensions of the blanks in the gears constituting the movable links of high kinematic joints; geometry and dimensions of the tooth surfaces, representing the geometric elements of kinematic joints (gearing).

The solution of the first group tasks is primarily given through the synthesis of the pitch configurations. It is partly presented in Part 1 of the study.

Later it will be discussed briefly the nature of the second group of tasks. Their formulation and adequate solution form the well-known in the theory of gearing "task for the synthesis of conjugate gearing" [9]. Here, it will be defined a simple formulation of the task for synthesis of spatial rack mechanisms, based on the second T. Olivier's principle [4]:

- It is given a structure scheme of spatial rack drive, for which one of the movable link $i=1$ is the rotating one and it represents gear with helical or curvilinear teeth. The second movable link $i=2$ realizes rectilinear translation and it is a rack with inclined (helical) teeth.

- Let us, assume, that one of the movable links, for example $i=1$, contains the preliminary defined active tooth surfaces $\Sigma_{1}^{(j)}(j=1,2)$ of the rotating link $i=1$ for its motion in both directions of rotation. These surfaces have to be smooth, without interruption and folds.

- The surfaces that are searched are: $\Sigma_{2}^{(j)}$, which should belong to the second movable link $i=2$ in such way that they have to form high kinematic joints $\left(\Sigma_{1}^{(j)}: \Sigma_{2}^{(j)}\right)$ with $\Sigma_{1}^{(j)}$, i.e. $\Sigma_{1}^{(j)}$ and $\Sigma_{2}^{(j)}$ have a constant contact in a line or a point. At least one high kinematic joint $\left(\Sigma_{1}^{(j)}: \Sigma_{2}^{(j)}\right)$ is configured, for this motions transformation upon a preliminary given law. 


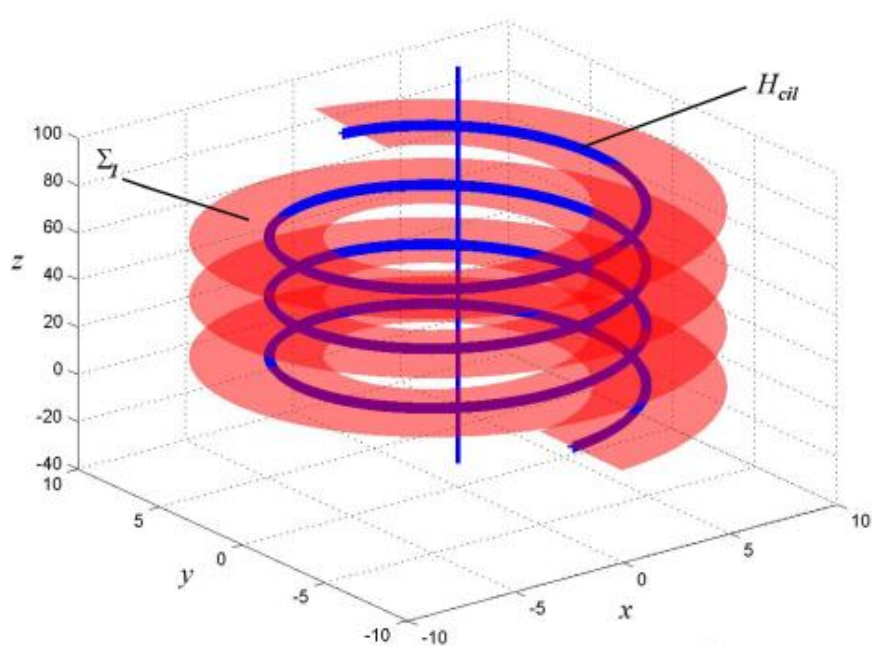

a)

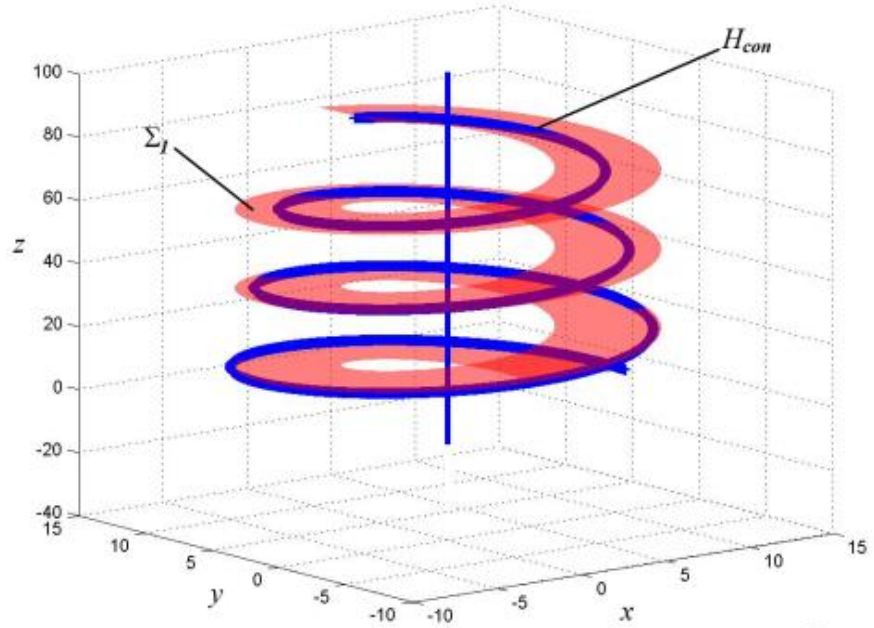

b)

Fig. 1. Ordinary helicoids: (a) Cylindrical helicoid $\Sigma_{1}$, considered as a locus of ordinary (cylindrical) helical lines $H_{\text {cil }}$; (b) Conic helicoid $\Sigma_{1}$, treated as a locus of ordinary (conic) helical lines $H_{\text {con }}$.

The described approach to the synthesis is applied in this study. It have to be noted, that other approaches to the synthesis of spatial rack drives, based on two main T. Olivier's principles, are not excluded.

It is known [10], that a helical motion is a complex motion, that consists of a rotation around fixed axis (axis of helical motion) with angular velocity vector $\bar{\omega}$ and 
rectilinear translation with velocity $\bar{V}$, parallel to the same axis. When parameter $p$ of the helical motion is $p=V / \omega=$ constant, [mm/rad], where $V$ and $\omega$ are the values of $\bar{V}$ and $\bar{\omega}$, it is called ordinary helical motion.

The line, formed from the ordinary helical motion of a point, is called an ordinary (cylindrical) helical line. It is placed on the straight circular cylinder with an axis coinciding with the axis of the helical motion, which defines its name.

The surface, generated at this ordinary helical motion of an arbitrary line $L$ is called an ordinary (cylindrical) helical surface (cylindrical helicoid). This surface can be considered as a locus of ordinary (cylindrical) helical lines (see Fig. 1a), i.e. these surfaces are characterized with the same helical parameter $p=$ constant.

It can be shown (as it is performed in Fig. 1b), that the ordinary helical surface can be treated as a locus of conic helical lines, having a constant axial parameter, which is different in size from the constant parameter $p$ of the ordinary (cylindrical) helical surface. In this case, as it will be shown further, the parameter of the helical surface is:

$$
p=p\left(p_{s}, p_{t}\right)=\text { constant },
$$

where $p_{s}$ is an axial parameter of an arbitrary conic helical line, placed on the cone with a constant axial angle at the tip $\delta_{1}=$ constant; $p_{t}$ - cross parameter depending on the angle $\delta_{1}$.

When an ordinary helical surface is a locus of the conic helical lines, which is characterized with two helical parameters $p_{s}$ and $p_{t}$, then this surface will be called ordinary conic helical surface or conic helical surface (conic helicoid). It is necessary to remind, that the axial parameter of the ordinary conic helical line is $p_{s}$, and for the conic helicoid $-p \neq p_{s}=$ constant. Further, under ordinary helical surfaces should be understood not only cylindrical but also conical helicoids.

The object of this study is a spatial rack drive, in which the rotating link is a gear with helical teeth and a constant axial pitch. Teeth of this type are generated by helical surfaces with constant helical parameter $[1,9,13]$. It will be noted that the axial parameter is the axial displacement of an arbitrary point from this helicoid, at its helical motions, corresponding to a rotation at an angle equal to $1 \mathrm{rad}$.

\subsection{KinEMATIC ANALYSiS OF THE ACTION SURFACE (MESH REgION) OF SPATIAL RACK DRIVE}

General equation of ordinary helicoid. It is known [1,14], that every helicoid can be generated by the helical motion of an arbitrary curve. Let, in this case, the curve is an arbitrary cross section of the ordinary helical surface $\Sigma_{1}$, defined in its own co-ordinate system $S_{w}\left(O_{w}, x_{w}, y_{w}, z_{w}\right)$ (see Fig. 2) with an equation in polar coordinates

$$
\bar{r}_{w}=r_{w}\left(\vartheta_{w}\right) \cos \vartheta_{w} \bar{i}_{w}+r_{w}\left(\vartheta_{w}\right) \sin \vartheta_{w} \bar{j}_{w}
$$




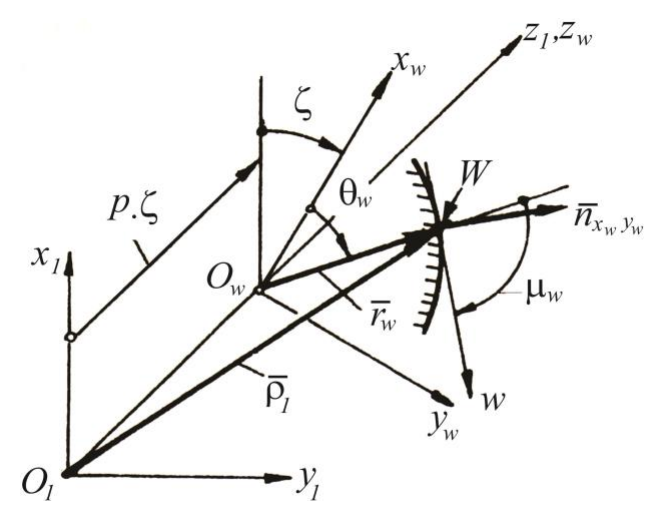

Fig. 2. Scheme of generation of ordinary helicoid $\Sigma_{1}$.

where $\vartheta_{w}$ is an angle parameter of the cross section of the helicoid $\Sigma_{1} ; \bar{i}_{w}, \bar{j}_{w}, \bar{k}_{w}-$ unit vectors of the right-handed co-ordinate system $S_{w}\left(O_{w}, x_{w}, y_{w}, z_{w}\right)$.

Then, in accordance with shown in Fig. 2, the vector equation of the helicoid $\Sigma_{1}$ is presented in its co-ordinate system $S_{1}\left(O_{1}, x_{1}, y_{1}, z_{1}\right)$ :

$$
\bar{\rho}_{1}=r_{w}\left(\vartheta_{w}\right) \cos \left(\vartheta_{w}+\zeta\right) \bar{i}_{1}+r_{w}\left(\vartheta_{w}\right) \sin \left(\vartheta_{w}+\zeta\right) \bar{j}_{1}+p \zeta \bar{k}_{1},
$$

where $\zeta$ is a second angle parameter of the helicoid $\Sigma_{1} ; p$ - helical parameter of $\Sigma_{1}$; $\bar{i}_{1}, \bar{j}_{1}, \bar{k}_{1}$ - unit vectors of the co-ordinate axes of the co-ordinate system $S_{1}\left(O_{1}, x_{1}, y_{1}, z_{1}\right)$. From the vector equation (2), the equation of the normal $\bar{n}_{1}$ at $\Sigma_{1}$ in its co-ordinate system is determined, as follows:

$$
\bar{n}_{1}=\frac{\partial \bar{\rho}_{1}}{\partial \vartheta_{w}} \times \frac{\partial \bar{\rho}_{1}}{\partial \zeta}=n_{1, x_{1}} \bar{i}_{1}+n_{1, y_{1}} \bar{j}_{1}+n_{1, z_{1}} \bar{k}_{1},
$$

where

$$
n_{1, x_{1}}=\left|\begin{array}{ll}
\frac{\partial y_{1}}{\partial \vartheta_{w}} & \frac{\partial y_{1}}{\partial \zeta} \\
\frac{\partial z_{1}}{\partial \vartheta_{w}} & \frac{\partial z_{1}}{\partial \zeta}
\end{array}\right|, \quad n_{1, y_{1}}=\left|\begin{array}{ll}
\frac{\partial z_{1}}{\partial \vartheta_{w}} & \frac{\partial z_{1}}{\partial \zeta} \\
\frac{\partial x_{1}}{\partial \vartheta_{w}} & \frac{\partial x_{1}}{\partial \zeta}
\end{array}\right|, \quad n_{1, z_{1}}=\left|\begin{array}{ll}
\frac{\partial x_{1}}{\partial \vartheta_{w}} & \frac{\partial x_{1}}{\partial \zeta} \\
\frac{\partial y_{1}}{\partial \vartheta_{w}} & \frac{\partial y_{1}}{\partial \zeta}
\end{array}\right|
$$

It is introduced an angle $\mu_{w}$, which the radius vector $\bar{r}_{w}$ of an arbitrary point $W$ of the cross section of the helicoid $\Sigma_{1}$ conclude with the positive direction of the tangent $w$ to the curve from the crossed section at the same point (see Fig. 2)

$$
\mu_{w}=\arctan \left(\frac{r_{w}}{d r_{w} / d \vartheta_{w}}\right)
$$


Then (3) is of the type:

(5) $n_{1, s_{1}}=\frac{r_{w}}{\sin \mu_{w}}\left[p \sin \left(\vartheta_{w}+\zeta+\mu_{w}\right) \bar{i}_{1}-p \cos \left(\vartheta_{w}+\zeta+\mu_{w}\right) \bar{j}_{1}+r_{w} \cos \mu_{w} \bar{k}_{1}\right]$.

After considering the vector equation (2), describing the elements in the matrices, for determining the scalar expressions of the projections of the normal vector $\bar{n}_{1, s_{1}}$ in its co-ordinate system, it can be written

$$
\begin{aligned}
\frac{\partial x_{1}}{\partial \vartheta_{w}} & =x_{1} \cot \mu_{w}-y_{1}, & & \frac{\partial x_{1}}{\partial \zeta}=-y_{1}, \\
\frac{\partial y_{1}}{\partial \vartheta_{w}} & =y_{1} \cot \mu_{w}+x_{1}, & \frac{\partial y_{1}}{\partial \zeta} & =x_{1}, \\
\frac{\partial z_{1}}{\partial \vartheta_{w}} & =0, & \frac{\partial z_{1}}{\partial \zeta} & =p .
\end{aligned}
$$

After substituting (6) in matrices from (3), it is obtained

$$
\begin{aligned}
& n_{1, x_{1}}=p\left(y_{1} \cot \mu_{w}+x_{1}\right) ; \\
& n_{1, y_{1}}=-p\left(x_{1} \cot \mu_{w}-y_{1}\right) ; \\
& n_{1, z_{1}}=r_{w}^{2} \cot \mu_{w}=\left(x_{1}^{2}+y_{1}^{2}\right) \cot \mu_{w} .
\end{aligned}
$$

The following analytical relation between the co-ordinates of an arbitrary point $W$ from the helicoid $\Sigma_{1}$ and the components of its normal vector $\bar{n}_{1}$ at the same point (written in the co-ordinate system $S_{1}\left(O_{1}, x_{1}, y_{1}, z_{1}\right)$ of $\left.\Sigma_{1}\right)$ is obtained, when the both sides of the equalities of (7) are multiplied with $\left(y_{1}\right)$ and $\left(-x_{1}\right)$, respectively and taking into account the third equality [1]:

$$
y_{1} n_{1, x_{1}}-x_{1} n_{1, y_{1}}=p n_{1, z_{1}} .
$$

Generating of the surface of action. It is known, that the mesh surface for all type of gear mechanisms represents the locus of the instantaneous contact lines of the conjugated active tooth surfaces of their movable links. Mesh region is that part of the surface of action, which is located between the surfaces, which limit the tips of the teeth of the two movable links of the mechanism.

This study is dedicated to the spatial rack drive, which kinematic scheme is illustrated in Fig. 3. The following transition matrices are written, when the co-ordinate system $S(O, x, y, z)$ - fixed to the frame and $S_{1}\left(O_{1}, x_{1}, y_{1}, z_{1}\right)$ - fixed to the link $i=1$ (corresponding to the same co-ordinate system $S_{1}$ from Fig. 2 for the parameter of meshing $\varphi_{1}=0$ ), are used

(9) $M_{S S_{1}}=\left\|\begin{array}{cccc}\cos \varphi_{1} & \sin \varphi_{1} & 0 & 0 \\ -\sin \varphi_{1} & \cos \varphi_{1} & 0 & 0 \\ 0 & 0 & 1 & 0 \\ 0 & 0 & 0 & 1\end{array}\right\|, \quad L_{S S_{1}}=\left\|\begin{array}{ccc}\cos \varphi_{1} & \sin \varphi_{1} & 0 \\ -\sin \varphi_{1} & \cos \varphi_{1} & 0 \\ 0 & 0 & 1\end{array}\right\|$. 


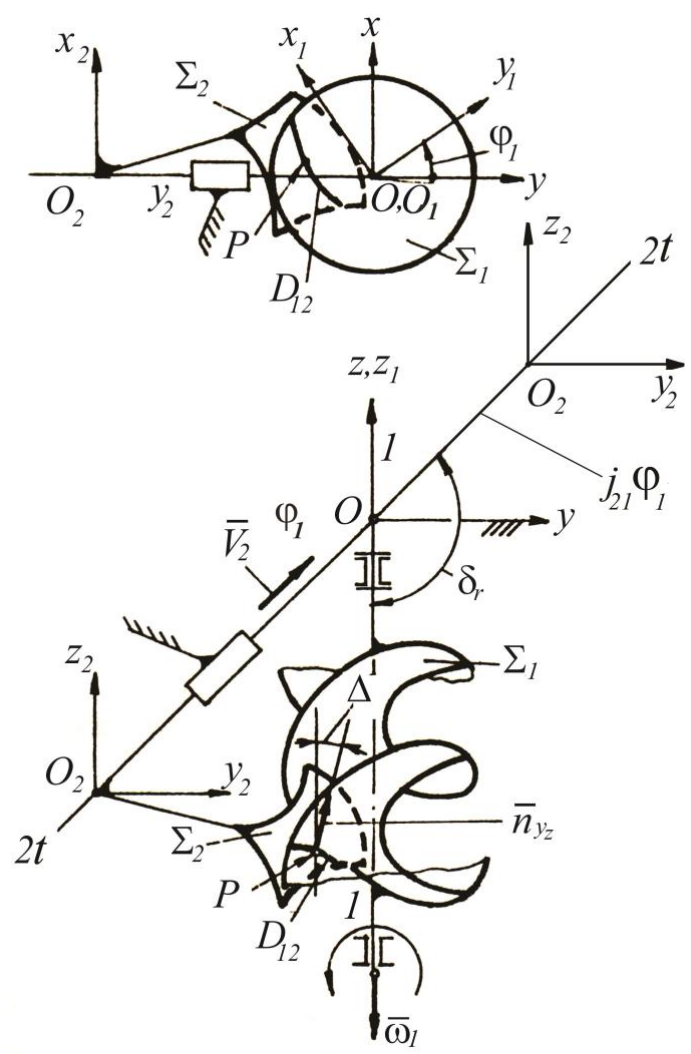

Fig. 3. Geometric-kinematic scheme of spatial motions transformation of type $(R \leftrightarrow T)$.

The equations of the helicoid $\Sigma_{1}$ and the normal vector $\bar{n}_{1}$ in the fixed space $S(O, x, y, z)$ have the following type:

$$
\begin{aligned}
& \bar{\rho}_{1, s}=r_{w} \cos (A) \bar{i}+r_{w} \sin (A) \bar{j}+p \cdot \zeta \bar{k}, \\
& \bar{n}_{1, s}=\frac{r_{w}}{\sin \mu_{w}}\left[p \sin (B) \bar{i}-p \cos (B) \bar{j}+r_{w} \cos \mu_{w} \bar{k}\right], \\
& A=\vartheta_{w}+\zeta-\varphi_{1}, \quad B=\vartheta_{w}+\zeta+\mu_{w}-\varphi_{1} .
\end{aligned}
$$

Analogically to what is shown with the expressions in (6), (7) and (8), from the vector equations (10), it is obtained

$$
y n_{1, x}-x n_{1, y}=p n_{1, z} .
$$

In the synthesis of gear mechanisms with kinematical conjugated surfaces $\Sigma_{1}$ and $\Sigma_{2}$, realizing a preliminary given law of motions transformation, it is essential to 
ensure their permanent contact. The kinematic condition for the existence of such continuous contact is the basic equation of meshing [1]

$$
\bar{n}_{1} \bar{V}_{12}=0 .
$$

From equation of meshing (12), when taking into account (10) and (11), it is written [14]

$$
\tan \Delta=\frac{n_{1, y}}{n_{1, z}}=\frac{p+j_{21} \cos \delta_{r}}{j_{21} \sin \delta_{r}}=\text { constant . }
$$

Equality (13) shows that the normal vector $\bar{n}_{1, s}$ in every point $P$ from the surface of action lies in the plane, parallel to the co-ordinate axis $O x$ and concluding a constant angle with the axis $O z$.

The expressions (10) and (13) will be used for the evaluation of the geometry of the mesh surface. Let point $P^{\prime}$ is determined by the parameters $\varphi_{1}^{\prime}, \vartheta_{w}^{\prime}$, $\zeta^{\prime}$, respectively $\bar{r}_{w}^{\prime}=\bar{r}_{w}\left(\vartheta_{w}^{\prime}\right)$ and $\mu_{w}^{\prime}=\mu_{w}\left(\vartheta_{w}^{\prime}\right)$. Hence, $P^{\prime}$ is a point from $\Sigma_{1}$ and for its radius-vector $\bar{\rho}_{1, s}^{\prime}$, as well for the normal vector $\bar{n}_{1, s}^{\prime}$ at the same point, it can be written

$$
\begin{aligned}
& \bar{\rho}_{1, s}^{\prime}=r_{w}^{\prime} \cos \left(A^{\prime}\right) \bar{i}+r_{w}^{\prime} \sin \left(A^{\prime}\right) \bar{j}+p \zeta \bar{k}, \\
& \bar{n}_{1, s}^{\prime}=\frac{r_{w}^{\prime}}{\sin \mu_{w}^{\prime}}\left[p \sin \left(B^{\prime}\right) \bar{i}-p \cos \left(B^{\prime}\right) \bar{j}+r_{w}^{\prime} \cos \mu_{w}^{\prime} \bar{k}\right], \\
& A^{\prime}=\vartheta_{w}^{\prime}+\zeta^{\prime}-\varphi_{1}, \quad B^{\prime}=\vartheta_{w}^{\prime}+\zeta^{\prime}+\mu_{w}^{\prime}-\varphi_{1}^{\prime},
\end{aligned}
$$

and therefore, the condition is satisfied

$$
\tan \Delta=-\frac{p \cos \left(B^{\prime}\right)}{r_{w}^{\prime} \cos \mu_{w}^{\prime}}
$$

Let link 1 is rotated around axis $1-1$ at angle $\Delta \varphi_{1}$. It will be shown that the point $P^{\prime \prime}$ is from $\Sigma_{1}$, which meets the following condition:

$$
\begin{aligned}
& \bar{\rho}_{1, s}^{\prime \prime}=\bar{\rho}_{1, s}\left(\vartheta_{w}^{\prime \prime}, \zeta^{\prime \prime}, \varphi_{1}^{\prime \prime}\right), \\
& \bar{n}_{1, s}^{\prime \prime}=\bar{n}_{1, s}\left(\vartheta_{w}^{\prime \prime}, \zeta^{\prime \prime}, \mu_{w}^{\prime \prime}, \varphi_{1}^{\prime \prime}\right),
\end{aligned}
$$

and correspondingly $\varphi_{1}^{\prime \prime}=\varphi_{1}^{\prime}+\Delta \varphi_{1}, \vartheta_{w}^{\prime \prime}=\vartheta_{w}^{\prime}, \mu_{w}^{\prime \prime}=\mu_{w}^{\prime}=\mu_{w}\left(\vartheta_{w}^{\prime}\right), \bar{r}_{w}^{\prime \prime}=\bar{r}_{w}^{\prime}=$ $\bar{r}_{w}\left(\vartheta_{w}^{\prime}\right), \zeta^{\prime \prime}=\zeta^{\prime}+\Delta \varphi_{1}$, is a point from the action surface. For the radius-vector $\bar{\rho}_{1, s}^{\prime \prime}$ of point $P^{\prime \prime}$ and the normal vector $\bar{n}_{1, s}^{\prime \prime}$ to $\Sigma_{1}$ in point $P^{\prime \prime}$ it can be written

$$
\begin{aligned}
& \bar{\rho}_{1, s}^{\prime \prime}=r_{w}^{\prime} \cos \left(A^{\prime \prime}\right) \bar{i}+r_{w}^{\prime} \sin \left(A^{\prime \prime}\right) \bar{j}+\left(p_{s} \zeta+p_{s} \Delta \varphi_{1}\right) \bar{k}, \\
& \bar{n}_{1, s}^{\prime \prime}=\frac{r_{w}^{\prime}}{\sin \mu_{w}^{\prime}}\left[p \sin \left(B^{\prime \prime}\right) \bar{i}-p \cos \left(B^{\prime \prime}\right) \bar{j}+r_{w}^{\prime} \cos \mu_{w}^{\prime} \bar{k}\right], \\
& A^{\prime \prime}=\vartheta_{w}^{\prime}+\zeta^{\prime}+\Delta \varphi_{1}-\varphi_{1}^{\prime}-\Delta \varphi_{1}, \\
& B^{\prime \prime}=\vartheta_{w}^{\prime}+\zeta^{\prime}+\Delta \varphi_{1}+\mu_{w}^{\prime}-\varphi_{1}^{\prime}-\Delta \varphi_{1} .
\end{aligned}
$$


When the first equations of the systems (14) and (17) are compared, it is established

$$
x^{\prime \prime}=x^{\prime}, \quad y^{\prime \prime}=y^{\prime}, \quad z^{\prime \prime}=z^{\prime}+p \Delta \varphi_{1} .
$$

In accordance with (13) and (15) it can be written

$$
-\frac{p \cos \left(\vartheta_{w}^{\prime \prime}+\zeta^{\prime \prime}+\mu_{w}^{\prime \prime}-\varphi_{1}^{\prime \prime}\right)}{r_{w}^{\prime \prime} \cos \mu_{w}^{\prime \prime}}=-\frac{p \cos \left(\vartheta_{w}^{\prime}+\zeta^{\prime}+\mu_{w}^{\prime}-\varphi_{1}^{\prime}\right)}{r_{w}^{\prime} \cos \mu_{w}^{\prime}},
$$

i.e.,

$$
\frac{n_{1, y}^{\prime}}{n_{1, z}^{\prime}}=\frac{n_{1, y}^{\prime \prime}}{n_{1, z}^{\prime \prime}}=\tan \Delta .
$$

Therefore, $P^{\prime \prime}$ is a point from mesh surface, i.e. $P^{\prime \prime}$ is a contact point between $\Sigma_{1}$ and $\Sigma_{2}$. In accordance with (18), if point $P^{\prime}\left(x^{\prime}, y^{\prime}, z^{\prime}\right)$ from $\Sigma_{1}$, belongs to the action surface, then all points from the line

$$
x=x^{\prime}, \quad y=y^{\prime}, \quad z=w, \quad w \in(-\infty,+\infty),
$$

also belong to this surface.

Hence, the mesh surface is a cylindrical surface with generatrices parallel to the axis $O z$. An arbitrary contact line $D_{12}$, (obtained for an arbitrary $\varphi_{1}=$ cosntant), represents a directrix of the surface of action. Analogically it is shown, that the active tooth surfaces $\Sigma_{2}$ of the gear rack are cylindrical surfaces as well $[4,14,15]$.

The characteristics obtained, as a result of the analysis realized here, are results, which can be treated not only as elements, extending the scientific knowledge of the theory of gearing, but also as analytical relations involved in the elaboration of mathematical models. These models serve the apparatus of geometric theory, which is oriented to the synthesis of rack drives. In other words, these characteristics are on the border of the two scientific fields - theory and geometry of gearing.

\section{SYNTHESIS OF SPATIAL RACK DRIVES, WHICH ROTATING LINK HAS CONIC LINEAR HELICOIDS}

The only case, that will be discussed below is about a rack mechanism with the most common conic linear helicoid- conic convolute helicoid $[4,15]$. From this case with simple substitutions, are obtained the cases, when the rotation link of the rack transmission is equipped with conic Archimedean or involute helicoids correspondingly, as its active tooth surfaces.

When synthesizing spatial rack mechanisms with linear contact, it is evident the necessity to control their quality in the whole mesh region. Such approaches to synthesis problems require an adequate mathematical model. The kinematic scheme of 


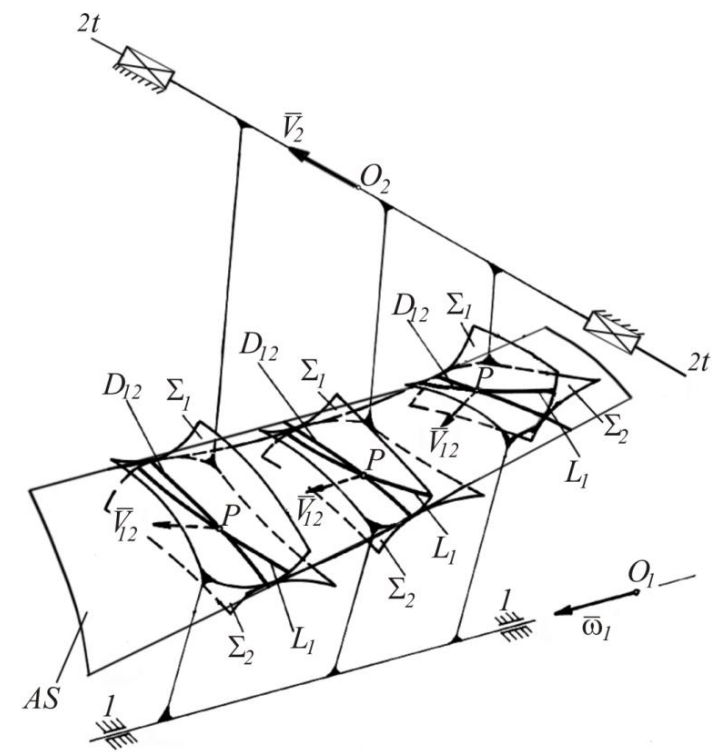

Fig. 4. Kinematic scheme of spatial rack drive with linear contact between tooth surfaces $\Sigma_{1}$ and $\Sigma_{2}$, synthesized using a mesh region (MR): $\Sigma_{i}-$ surface of flank of links $(i=1,2)$; $\bar{\omega}_{1}$ - rotation velocity of link $i=1 ; \bar{V}_{2}$ - velocity of rectilinear translation of link $i=2 ; A S$ - action surface; $D_{12}$ - contact line; $L_{1}$ - longitudinal line of $\Sigma_{1}$.

such mechanism is shown in Fig. 4. A mathematical model, based on a mesh region is not a universal one.

The reason is that the specific geometrical and kinematic characteristics of the mesh region depend on its position in space and the geometric characteristics of the instrumental surface $\Sigma_{J}$, which generates the flanks $\Sigma_{i}(i=1,2)$.

This mathematical model is based on the second Olivier's principle and the link, performing rotation and having surfaces $\Sigma_{1}$ is chosen as a generating link. Generated surfaces $\Sigma_{2}$ belong to link 2, which realizes rectilinear translation.

The synthesis of spatial rack drives, based on the second Olivier's principle, involves solving two main tasks:

- Synthesis of the tooth surfaces $\Sigma_{1}$ of the rotating movable link, which are identical to the instrumental surfaces $\Sigma_{J}$. Solving this mathematical problem provides the technology of manufacture the rotating link of the rack drive.

- Synthesis of the mesh region and definition of the dimensions and location of the mesh region on the surface of action. This task realizes the generation of the active tooth surfaces $\Sigma_{2}$ of the movable link of the rack mechanisms, which perform motion translation. 
The synthesis of these types of mechanical transmissions, in accordance with the second Olivier's principle, leads to solving two main tasks: synthesis of the active tooth surfaces of the basic (instrumental) movable link - conic worm and synthesis of surface of action/mesh region of the studied mechanism. The active tooth surfaces of the basic link are conic linear helicoids.

\subsection{GENERATING ACTIVE TOOTH SURFACES OF THE BASIC MOVABLE LINK}

The helicoids and especially the linear helicoids are widely used as active tooth surfaces of spatial gears with crossed axes, in both working meshing conditions and instrumental meshing conditions. Such choice of the active tooth surfaces for these gears is determined by the technological manufacturing, especially when their synthesis is realized in accordance with the second Oliver's principle [1,9]. In most cases, these surfaces are the only alternative, as it is the manufacture of cylindrical worm gear and Spiroid and Helicon gears.

Figure 5 shows the generation of right-handed conic convolute helicoids $\Sigma_{1}^{(j)}$ $(j=1,2)$, determined by different geometrical characteristics of the helical teeth (treads) of the particular gear mechanism. The process of helical surfaces generation

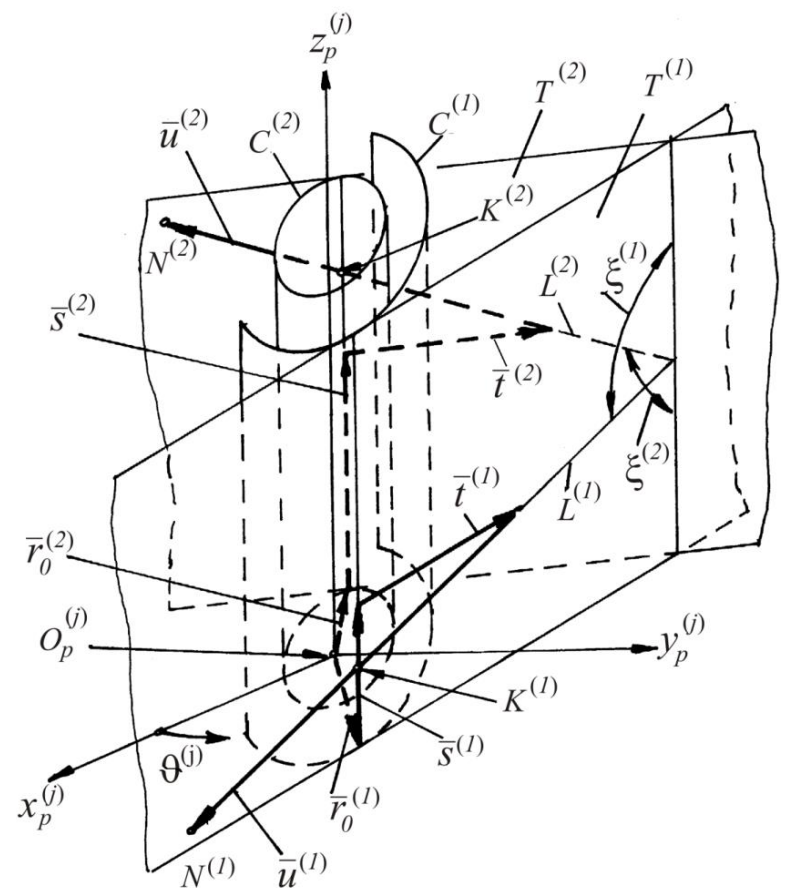

Fig. 5. Conic convolute helicoids generation. 
is considered in fixed co-ordinate system $S_{f}\left(O_{f}, x_{f}, y_{f}, z_{f}\right)$ and it is as follows. The generatrix $\Sigma^{(j)}$ does not cross the axis $O_{p}^{(j)} z_{p}^{(j)}$, which coincides with the geometric axis of the gear. $\Sigma^{(j)}$ and $O_{p}^{(j)} z_{p}^{(j)}$ conclude an angle $0,5 \pi<\xi^{(j)}<\pi$. The line $L^{(j)}$ belongs to plane $T^{(j)}$, which is tangential to the directed circle cylinder $C^{(j)}$. The generation of the conic convolute helicoid $\Sigma_{1}^{(j)}$ from the line $L^{(j)}$ is realized by two generatrix motions: axial helical motion, along the longitudinal axis $O_{p}^{(j)} z_{p}^{(j)}$ with parameter $p_{s}^{(j)}=$ constant; crossed helical motion in the plane $T^{(j)}$, perpendicular to the axis $O_{p}^{(j)} z_{p}^{(j)}$ with parameter $p_{t}^{(j)}=$ constant. Here, it should be noted that $\Sigma_{1}^{(1)}$ is the conic convolute helical surface, that is turned along the positive direction of the axis $O_{p}^{(1)} z_{p}^{(1)}$, and $\Sigma_{1}^{(2)}$ is the helicoid, turned along the negative direction of the $O_{p}^{(2)} z_{p}^{(2)}$.

The vector equation of the conic convolute helical surface $\Sigma_{1}^{(1)}$, in accordance with Fig. 5, has the form $[9,15]$

$$
\bar{\rho}_{1}^{(j)}=\bar{r}_{0}^{(j)}+\bar{s}^{(j)}+\bar{t}^{(j)}+\bar{u}^{(j)},
$$

where $\bar{\rho}_{1}^{(j)}$ is a radius-vector of point $N^{(j)}$, that belongs to the conic convolute helicoid $\Sigma_{1}^{(j)} ; \bar{r}_{0}^{(j)}$ - radius-vector of the directed cylinder $C^{(j)} ; \vartheta^{(j)}, u^{(j)}$ - co-ordinates of the helical surface $\Sigma_{1}^{(j)} ; s^{(j)}=p_{s}^{(j)} \vartheta^{(j)}$ - axial motion of the generatrix $L^{(j)}$; $t^{(j)}=p_{t}^{(j)} \vartheta^{(j)}$ - crossed displacement (tangential to the directed cylinder $C^{(j)}$ ) of the generatrix line $L^{(j)}$. If (20) is written in co-ordinate system $S_{p}^{(j)}$, then it is obtained

$$
\begin{aligned}
& x_{p}^{(j)}=r_{0}^{(j)} \cos \vartheta^{(j)} \pm\left(u^{(j)} \sin \xi^{(j)}-p_{t}^{(j)} \vartheta^{(j)}\right) \sin \vartheta^{(j)}, \\
& y_{p}^{(j)}=r_{0}^{(j)} \sin \vartheta^{(j)} \mp\left(u^{(j)} \sin \xi^{(j)}-p_{t}^{(j)} \vartheta^{(j)}\right) \cos \vartheta^{(j)}, \\
& z_{p}^{(j)}=p_{s}^{(j)} \vartheta^{(j)} \pm u^{(j)} \cos \xi^{(j)} .
\end{aligned}
$$

For the equation systems (21), the upper signs and $j=1$ refer to the $\Sigma_{1}^{(1)}$, and the lower and $j=2$ refer to $\Sigma_{1}^{(2)}$.

Substituting in (21)

$$
R_{0}^{(j)}=u^{(j)}-\frac{p_{t}^{(j)} \vartheta^{(j)}}{\sin \xi^{(j)}} \quad \text { and } \quad p^{(j)}=p_{s}^{(j)} \pm p_{t}^{(j)} \cot \xi^{(j)}
$$

it is obtained

$$
\begin{aligned}
& x_{p}^{(j)}=r_{0}^{(j)} \cos \vartheta^{(j)} \pm R_{0}^{(j)} \sin \xi^{(j)} \sin \vartheta^{(j)} \\
& y_{p}^{(j)}=r_{0}^{(j)} \sin \vartheta^{(j)} \mp R_{0}^{(j)} \sin \xi^{(j)} \cos \vartheta^{(j)} \\
& z_{p}^{(j)}=p^{(j)} \vartheta^{(j)} \pm R_{0}^{(j)} \cos \xi^{(j)}
\end{aligned}
$$


Equation (23) represents the conic convolute helical surface $\Sigma_{1}^{(j)}$ as a cylindrical one with helical parameter $p^{(j)}=$ constant and co-ordinates $\vartheta^{(j)}$ and $R_{0}^{(j)}$. The point $K^{(j)}$ is the accounting origin of co-ordinate $R_{0}^{(j)},\left(K^{(j)}\right.$ is a point of intersection of $L^{(j)}$ and the generatrix of the directed cylinder $C^{(j)} ; C^{(j)}$ and plane $T^{(j)}$ are contacting in this generatrix $\left.L^{(j)}\right)$. The point $K^{(j)}$ is considered to be a point from the directed helical line $\bar{\rho}_{0}^{(j)}=\bar{\rho}_{0}^{(j)}\left(\vartheta^{(j)}\right)$ on $C^{(j)}$.

\subsection{SingULAR POINTS ON THE CONIC CONVOLUTE HELICOIDS}

Here, is presented a study of the conditions, under which in the process of meshing of the kinematic conjugated surfaces $\Sigma_{1}$ and $\Sigma_{2}$ of the spatial rack drives, the contact points are transformed into singular points. The study is based on the theoretical approach, offered by Prof. F. Litvin [1-3]. According to it, the singular points, in the most common case, are those points between $\Sigma_{1}$ and $\Sigma_{2}$, for which the condition "the relative velocity on one of the contacting surfaces (written in the fixed co-ordinate systems) is zero" is fulfilled.

Depending on the normal vector $\bar{n}_{i}$ at the common contact point of the meshed surfaces $\Sigma_{1}$ and $\Sigma_{2}$ two types of singular points exist:

- Singular points of first order, called ordinary nodes, if $\bar{n}_{i} \neq \overline{0}$ and consequently $\dot{\bar{n}}_{r, i}=\overline{0}\left(\dot{\bar{n}}_{r, i}\right.$ is the relative velocity vector of the tip of the normal vector $\left.\bar{n}_{i}\right)$.

It is possible to exist ordinary contact points (singular point of first order) on the conjugated tooth surfaces of spatial drives. These points are tangent points or points of intersection of the contact lines. Their existence, in the mesh region of spatial gears, should be limited or completely eliminated, since they increase specific friction, reduce lubrication and heat transfer, and as a result, they decrease the loading capacity of the synthesized gear.

- Singular points of second order, called points of undercutting, when $\bar{n}_{i}=\overline{0}$.

Singular points of second order should be eliminated from the mesh region, since they are points of undercutting for the instrumental meshing, or interferential points in the cases of working meshing of the conjugated tooth surfaces of the synthesized drives. The undercutting, as a rule, makes the tooth thinner and the gears strength becomes worse.

In order to eliminate the "undercutting" from the active tooth surfaces of the gears and the devices, representing parts of the conic linear helicoids, it is necessary to define conditions for appearance of the singular points (undercutting points) on them in the process of their generation. As it has been already shown, the conic linear 
helicoids $\Sigma_{1}^{(j)}$ (in particular conic convolute helical surface) have the form

$$
\bar{\rho}_{1}^{(j)}=\bar{\rho}_{1}^{(j)}\left(u^{(j)}, \vartheta^{(j)}\right) .
$$

Then, the normal vector $\bar{n}_{1}^{(j)}$ to the $\Sigma_{1}^{(j)}$ in arbitrary point $N^{(j)}$ is determined by the equality

$$
\bar{n}_{1}^{(j)}=\frac{\partial \bar{\rho}_{1}^{(j)}}{\partial u^{(j)}} \times \frac{\partial \bar{\rho}_{1}^{(j)}}{\partial \vartheta^{(j)}},
$$

where $\frac{\partial \bar{\rho}_{1}^{(j)}}{\partial u^{(j)}}$ and $\frac{\partial \bar{\rho}_{1}^{(j)}}{\partial \vartheta^{(j)}}$ are vectors at point $N_{1}^{(j)}$ from the conic helical surface $\Sigma_{1}^{(j)}$, that are tangential to the co-ordinate lines $u^{(j)}=$ constant and $\vartheta^{(j)}=$ constant. It is known [9], that the point $N^{(j)}$ from $\Sigma_{1}^{(j)}$ is an undercutting point, if for this point the normal vector $\bar{n}_{1}^{(j)}$ cannot be defined, i.e., if the following condition is fulfilled:

$$
\bar{n}_{1}^{(j)}=\overline{0} .
$$

Then from (21), the projections of the normal vector $\bar{n}_{1}^{(j)}$ in the co-ordinate system $S_{p}^{(j)}$ are written of the form

$$
\begin{aligned}
& n_{1, x_{p}}^{(j)}=\mp h^{(j)} \sin \xi^{(j)} \cos \vartheta^{(j)}-\left(u^{(j)} \sin \xi^{(j)}-p_{t}^{(j)} \vartheta^{(j)}\right) \cos \xi^{(j)} \sin \vartheta^{(j)} \\
& n_{1, y_{p}}^{(j)}=\mp h^{(j)} \sin \xi^{(j)} \sin \vartheta^{(j)}+\left(u^{(j)} \sin \xi^{(j)}-p_{t}^{(j)} \vartheta^{(j)}\right) \cos \xi^{(j)} \cos \vartheta^{(j)} \\
& n_{1, z_{p}}^{(j)}=\left(u^{(j)} \sin \xi^{(j)}-p_{t}^{(j)} \vartheta^{(j)}\right) \sin \xi^{(j)}
\end{aligned}
$$

From (26) it is evident, that the condition (25) is never satisfied for the conic convolute helicoid, since the conic convolute parameter of distribution is $[4,12]$

$$
h^{(j)}=p^{(j)}+r_{0}^{(j)} \cot \xi^{(j)} \neq 0 .
$$

\subsection{SYNTHESIS OF THE SURFACE OF ACTION/MESH REGION OF THE CONIC CONVOLUTE RACK DRIVES}

The action surface, considered as a locus of the lines of the tooth contact in the fixed space, defines conjugate tooth surfaces of the second movable link - gear rack. Three computer programs are written with analogical (typified) structure and organization of the calculating process, based on the realized study and on the developed algorithms for synthesis of three types spatial rack drives - cylindrical with cylindrical linear rotating helicoids, face (with face linear rotating helicoids) and with conic 


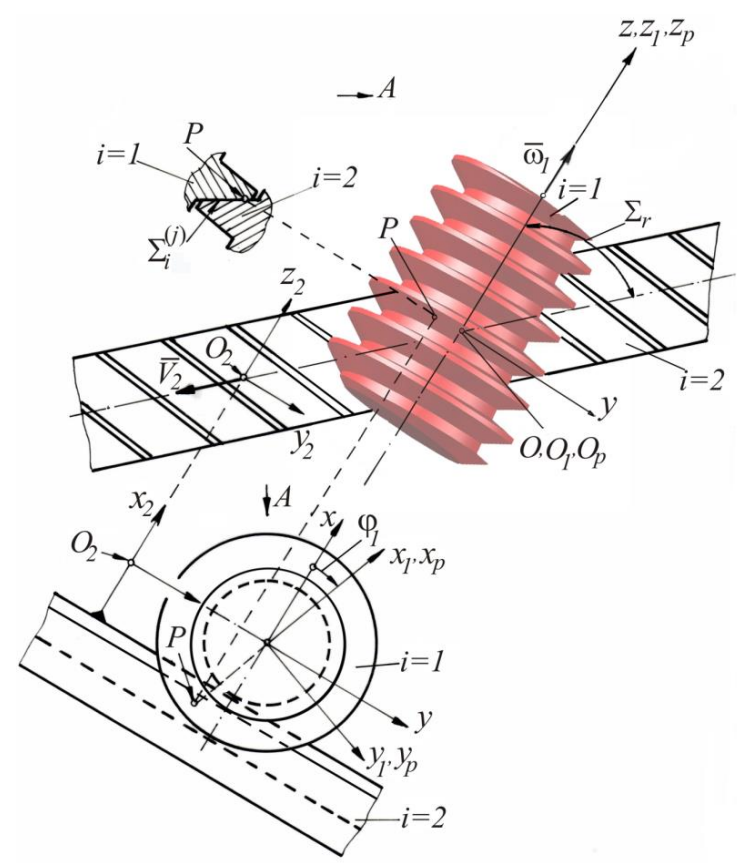

Fig. 6. Geometric-kinematic scheme of spatial rack drive, having rotating linear conic helicoids.

(with conic linear rotating helicoids). The developed programs illustrate the cases of the spatial conic, cylindrical and face rack drives synthesis. The study offered here is consistent with the symbols, shown in Fig. 6.

Conic convolute rack mechanism. The equations (23) of the linear helicoid $\Sigma_{1}^{(j)}$ are presented as

$$
\begin{aligned}
& x_{p}^{(j)}=r_{0}^{(j)} \cos \vartheta^{(j)} \pm U^{(j)} \sin \vartheta^{(j)}, \\
& y_{p}^{(j)}=r_{0}^{(j)} \sin \vartheta^{(j)} \mp U^{(j)} \cos \vartheta^{(j)}, \\
& z_{p}^{(j)}=p^{(j)} \vartheta^{(j)} \pm U^{(j)} \cot \xi^{(j)},
\end{aligned}
$$

where

$$
U^{(j)}=R_{0}^{(j)} \sin \xi^{(j)}=u^{(j)} \sin \xi^{(j)}-p_{t}^{(j)} \vartheta^{(j)}, \quad p^{(j)}=p_{s}^{(j)} \pm p_{t}^{(j)} \cot \xi^{(j)} .
$$

The set of equations (28) describes the conic convolute helicoid as a cylindrical one with helical parameter $p^{(j)}$. The normal vector in an arbitrary point of $\Sigma_{1}^{(j)}$ is described by equations system 


$$
\begin{aligned}
& n_{1, x_{p}}^{(j)}=\mp h^{(j)} \sin \xi^{(j)} \cos \vartheta^{(j)}-U^{(j)} \cos \xi^{(j)} \sin \vartheta^{(j)}, \\
& n_{1, y_{p}}^{(j)}=\mp h^{(j)} \sin \xi^{(j)} \sin \vartheta^{(j)}+U^{(j)} \cos \xi^{(j)} \cos \vartheta^{(j)}, \\
& n_{1, z_{p}}^{(j)}=U^{(j)} \sin \xi^{(j)} .
\end{aligned}
$$

It is known [9,15], that conic helicoids $\Sigma^{(j)}(j=1,2)$ are cylindrical helical surfaces with constant helical parameter $p^{(j)}=p_{s}^{(j)} \pm p_{t}^{(j)} \cot \xi^{(j)}=$ constant.

Hence, for these surfaces is valid the equation of meshing [14]

$$
\tan \Delta=\frac{n_{1, y}}{n_{1, z}}=\frac{p+j_{21} \cos \delta_{r}}{j_{21} \sin \delta_{r}}=\text { constant }
$$

Then, the action surface of the conic convolute helicoid is described by using the equations (28), (29) (written in the fixed co-ordinate system $S(O, x, y, z)$ ), and the analytical type (30) of equation of meshing (see Fig. 7), i.e.,
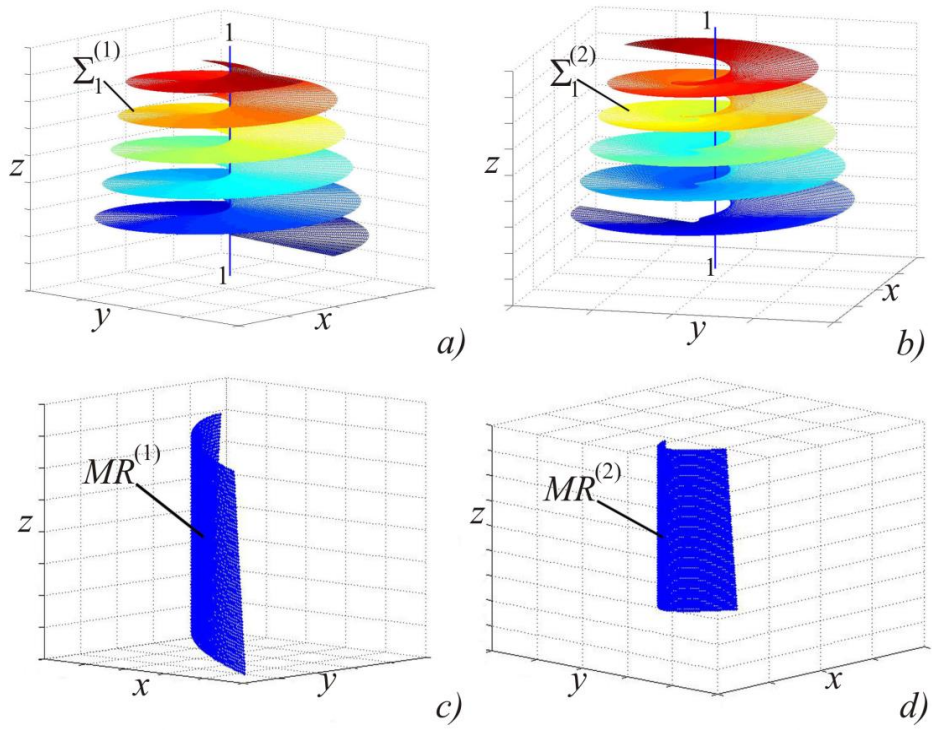

Fig. 7. Spatial conic convolute mechanism with velocity ratio $j_{21}=2.29$; number of the teeth $z_{1}=1$ : a) conic convolute worm $\Sigma_{1}^{(1)} \Rightarrow \xi^{(1)}=98^{\circ}, r_{0}^{(1)}=0.08 \mathrm{~mm} ; u^{(1)} \in[0,10]$, $\vartheta^{(1)} \in[0,5 \pi]$; b) $\Sigma_{1}^{(2)} \Rightarrow \xi^{(2)}=120^{\circ}, r_{0}^{(2)}=0.94 \mathrm{~mm} ; u^{(2)} \in[0,10], \vartheta^{(2)} \in[0,5 \pi]$; c) region of mesh $M R^{(1)}$; d) $M R^{(2)}$. 


$$
\begin{aligned}
& x^{(j)}=r_{0}^{(j)} \cos \theta^{(j)} \pm U^{(j)} \sin \theta^{(j)}, \\
& y^{(j)}=r_{0}^{(j)} \sin \theta^{(j)} \mp U^{(j)} \cos \theta^{(j)}, \\
& z^{(j)}=p^{(j)} \vartheta^{(j)} \pm U^{(j)} \cot \xi^{(j)} \\
& \frac{\mp h^{(j)} \sin \xi^{(j)} \sin \theta^{(j)}+U^{(j)} \cos \xi^{(j)} \cos \theta^{(j)}}{U^{(j)} \sin \xi^{(j)}}=\frac{p^{(j)}-j_{21} \cos \Sigma_{r}}{j_{21} \sin \Sigma_{r}},
\end{aligned}
$$

where $\theta^{(j)}=\vartheta^{(j)}+\varphi_{1}, U^{(j)}=u^{(j)} \sin \xi^{(j)}-p_{t}^{(j)} \vartheta^{(j)} \neq 0, p^{(j)}=p_{s}^{(j)} \pm p_{t}^{(j)} \cot \xi^{(j)}$, $h^{(j)}=p^{(j)}+r_{0}^{(j)} \cot \xi^{(j)}$. The conjugated with $\Sigma_{1}^{(j)}(j=1,2)$ tooth surfaces $\Sigma_{2}^{(j)}$ $(j=1,2)$ of the tooth rack $i=2$ are obtained from the system (31), after writing it in the co-ordinate system $S_{2}\left(O_{2}, x_{2}, y_{2}, z_{2}\right)$, firmly connected with link $i=2$ :

$$
\begin{aligned}
& x_{2}^{(j)}=r_{0}^{(j)} \cos \theta^{(j)} \pm U^{(j)} \sin \theta^{(j)}, \\
& y_{2}^{(j)}=r_{0}^{(j)} \sin \theta^{(j)} \mp U^{(j)} \cos \theta^{(j)}+j_{21} \varphi_{1} \sin \Sigma_{r}, \\
& z_{2}^{(j)}=p^{(j)} \vartheta^{(j)} \pm U^{(j)} \cot \xi^{(j)}+j_{21} \varphi_{1} \cos \Sigma_{r}, \\
& \frac{\mp h^{(j)} \sin \theta^{(j)}+U^{(j)} \cot \xi^{(j)} \cos \theta^{(j)}}{U^{(j)}}=\frac{p^{(j)}-j_{21} \cos \Sigma_{r}}{j_{21} \sin \Sigma_{r}} .
\end{aligned}
$$

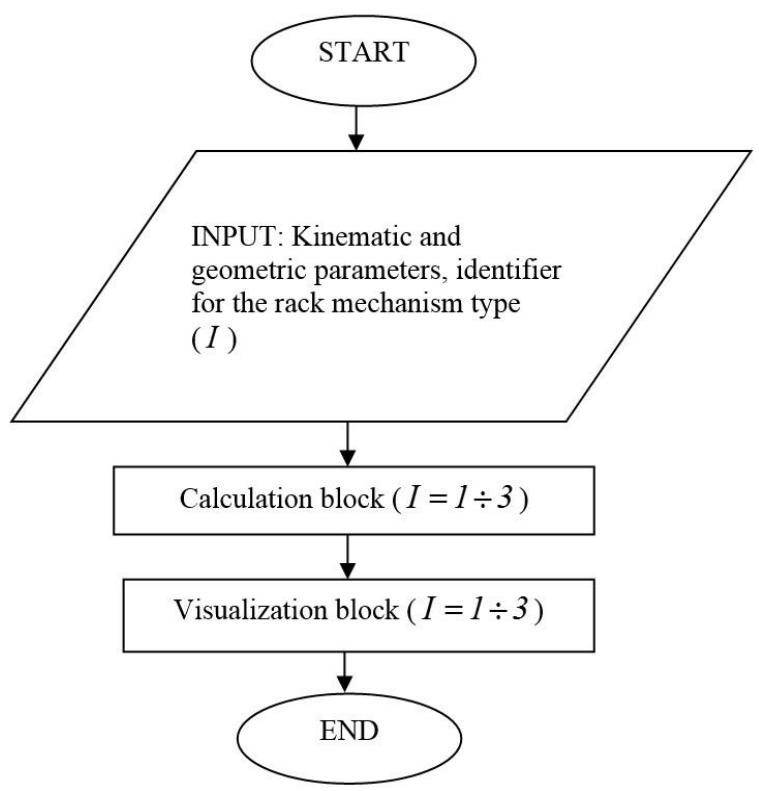

Fig. 8. Common block-scheme. 


\section{COMPUTER PROGRAMS FOR VISUALIZATION OF THE SYNTHESIS OF SPATIAL RACK DRIVES}

Three computer programs with similar (typified) structure and organization of their calculation process are written, on the basis of this study (which is part of the presented in $[4,15]$ ) and on the basis of the defined algorithms for synthesis of three types of spatial rack mechanisms - cylindrical (with cylindrical linear and curvilinear rotating helicoids), face (with linear rotating helicoids) and conic ones (with conic linear rotating helicoids). This product is briefly presented here.

The program has the characteristics of the "directed search" method for synthesis, but also this program realizes the visual study of the character of the geometry of active geometric elements $\Sigma_{i}^{(j)}(i=1,2, j=1,2)$ of the kinematic joints $\left(\Sigma_{1}^{(j)}\right.$ : $\left.\Sigma_{2}^{(j)}\right)$, by which the defined law for transformation of type $(R \leftrightarrow T)$ is realized. The program is written in MATLAB [17]. It contains description and declaration of input parameters and equation systems. The basic characteristics of the spatial rack mechanisms are obtained by solving these systems. The content of the programs is illustrated in Figs. 8, 9 and 10.

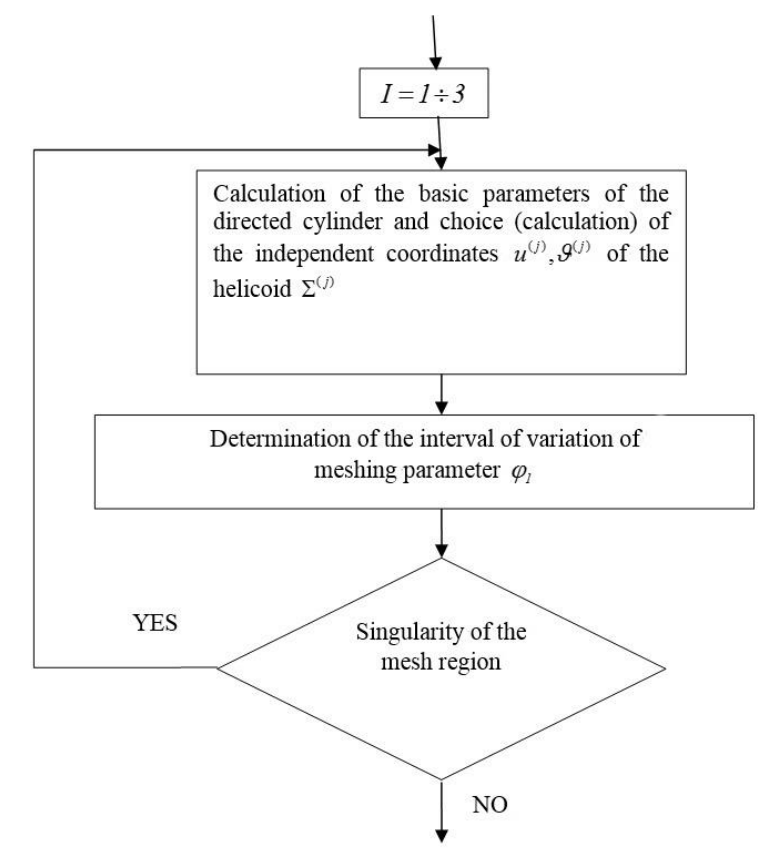

Fig. 9. Calculation block: $u^{(j)}, \vartheta^{(j)}$-independent co-ordinates of the linear $\Sigma^{(j)}(j=1$ for the low-side helicoids, $j=2$ for the high-side helicoids). 


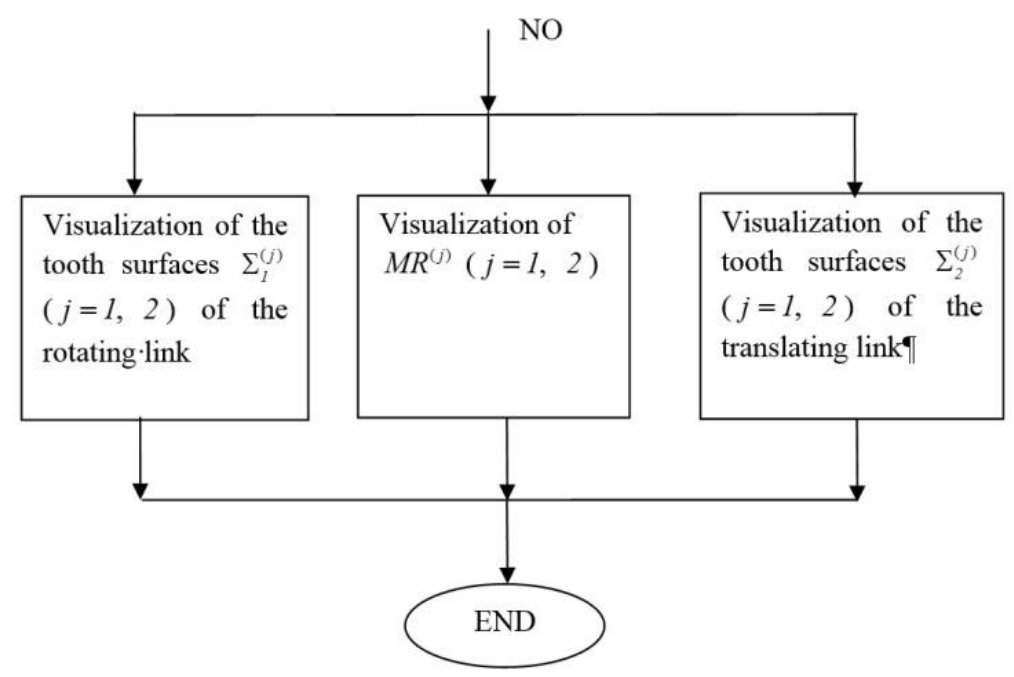

Fig. 10. Visualization block: $M R^{(j)}(j=1,2)-$ mesh regions of the synthesized rack mechanism.

The input parameters of the programs are:

- Geometric parameters: shaft angle $\Sigma_{r}$ [grad] (for face rack mechanisms $\Sigma_{r}=90^{\circ}$ ); crossed angle $\delta_{r}$ [grad]; radius of the pitch circle of the rotating link $-r_{1}[\mathrm{~mm}]$; an obtuse angle between linear generatrix of the helicoid and axis of the rotating link $\xi^{(j)}$ [deg]; tooth module $m$ [mm]; axial helical parameter $p_{s}[\mathrm{~mm} / \mathrm{rad}]$ (for cylindrical rack drive); crossed tangential parameter $p_{t}[\mathrm{~mm} / \mathrm{rad}]$ (for face rack drive); number of helicoids $\Sigma_{1}^{(j)}$ of the rotating link $i=1$;

- Kinematic parameters: velocity ratio $j_{12} / j_{21}[\mathrm{~mm} / \mathrm{rad}] /[\mathrm{mm} / \mathrm{rad}]$; parameter of meshing $\varphi_{1}[\mathrm{deg}]$;

- Identifier for the convolute rack mechanism (cylindrical, face, conic) $I=1$; for Archimedean rack mechanism (cylindrical, face, conic) $-I=2$; for involute rack mechanism (cylindrical, face, conic) $-I=3$;

Diagrammatically, the calculation block of the program is organized in accordance with Fig. 9 and the visualization block is shown in Fig. 10.

The studied rack drives can be synthesized and visualized by using the described computer programs and AutoCAD [17]. One specific representative of the studied mechanical motion transformer is illustrated in Fig. 11. 


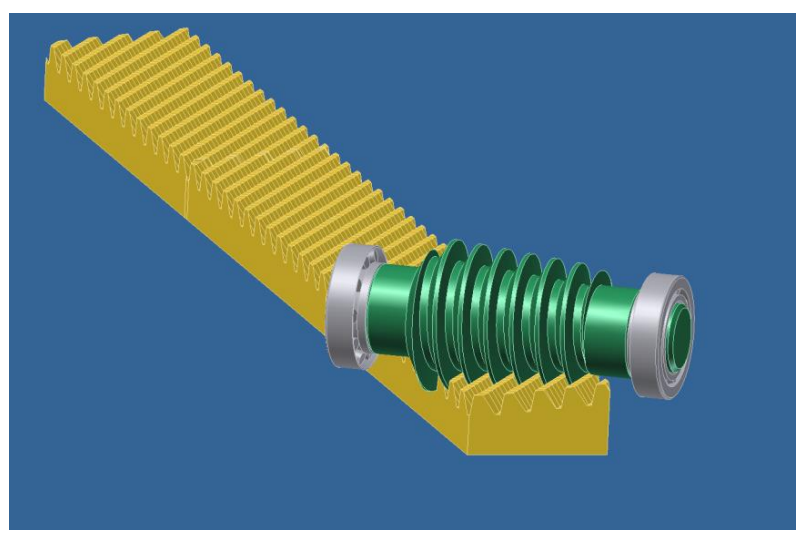

Fig. 11. Spatial conic rack mechanism.

\section{CONCLUSiON}

The kinematic theory illustrated here, that treats the geometry of gearing of the conic convolute rack drives, is just a part of the studies, realized by authors in this field. From this theory, an algorithms, for the synthesis of convolute (presented in this article), Archimedean and involute conic rack drives, are obtained. Special cases of the mentioned above rack mechanisms, are the cylindrical and the face ones, when the rotating link of the drive is a convolute, Archimedean (see Fig. 11) and involute, cylindrical or face worm, or a worm, whose threads are circle helicoids [4,15]. The shown algorithms are based on the studies, described in Part 1, which are the forming elements of the theory of gearing of the treated class spatial transmissions.

\section{REFERENCES}

[1] Litvin, F. Theory of Gearing, Moskow, Nauka, 1968, 584 (in Russian).

[2] Litvin, F. Theory of Gearing. NASA Reference Publication 1212, AVSCOM Technical Report 88-C-035, Washington, US Government Printing Office, 1989, 470.

[3] Litvin, F. Gearing Geometry and Applied Theory, New Jersey 07632, PTR Prentice Hall, A Paramount Communication Company, Englewood Eliffs, 1994, 724.

[4] Abadjieva, E. Mathematical Models of the Kinematic Processes in Spatial Rack Mechanisms and their Application, Ph. D Thesis, Bulgaria, Sofia, Institute of Mechanics - BAS, 2010, 165.

[5] Spur, G., Th. Stoferle. Handbook of Cutting Material Technologies, Book 2, Moscow, Ed. Mechanical Engineering Publishing House, 1985, 688 (in Russian).

[6] SAARI, O. The Mathematical Background of Spiroid Gears, Detroit, 'Industrial Mathematical Series", No 7, 1956, 131-144. 
[7] Ginzburg, E., N. Golovanov, N. Firun, N. Hlebsky. Handbook Mashinostroene, Leningrad, 1980, 416.

[8] Watanabe, M., M. Maki. A Study on New Type WN Rack and Pinion, Proceedings of The $2^{\text {nd }}$ Int. Conf. "Power Transmissions 2006", Balkan Association of Power Transmission, Faculty of Technical Sciences, Novi Sad 2006, 27 - 32.

[9] Abadjiev, V. Gearing Theory and Technical Applications of Hyperboloid Mechanisms, D.Sc. Thesis, Sofia, Institute of Mechanics, Bulgarian Academy of Sciences, 2007, 309 (In Bulgarian)

[10] Luykshin, V. Theory of Helical Surfaces in the Designed of the Cutting Devices, Moscow, Mechanical engineering, 1968, 371.

[11] Abadjiev, V., K. Minkov. On the Geometry of Helical Surfaces of Spiroid Transmissions. Journal of Theoretical and Applied Mechanics, 11 (1981), No. 2, 17-27.

[12] AbAdjIEV, V. On the Geometry of Convolute Spiroiden Helicoid, $4^{\text {th }}$ National Congress of Theoretical and Applied Mechanics, Varna, 14-18 September 1981, Book 1, Publishing House of BAS, Sofia, 1981, 158-163.

[13] Abadjiev, V. On the Synthesis and Analysis of Spiroid Gears, Ph. D. Thesis, Sofia, 1984, 158 (in Bulgarian)

[14] Kovatchev, G., V. Abadjiev. On the Synthesis of Spatial Rack Mechanisms, Proc. 6-th National Congress of Theor. and Appl. Mech., Varna, 1, 1990, 35-38, (in Russian).

[15] Abadjieva, E. Spatial Rack Drives. Mathematical Modelling for Synthesis, VDM Verlag Dr. Müller e.K., 2011, 72.

[16] Matlab, MathWorks, Release2006B.

[17] AutoCAD Inventor Professional Suite 2010. 